\title{
Um panorama de Teses e Dissertações brasileiras sobre Mundos Virtuais 3D na educação
}

\author{
Aliane Loureiro Krassmann ${ }^{1}$, Andressa Falcade ${ }^{2}$, Rafaela Ribeiro Jardim ${ }^{1}$, \\ Roseclea Duarte Medina ${ }^{2}$, Magda Bercht ${ }^{1}$ \\ ${ }^{1}$ Programa de Pós-Graduação em Informática na Educação (PPGIE) - Universidade \\ Federal do Rio Grande do Sul (UFRGS) - Porto Alegre - RS - Brasil \\ ${ }^{2}$ Programa de Pós-Graduação em Informática (PPGI) - Universidade Federal de Santa \\ Maria (UFSM) - Santa Maria - RS - Brasil \\ \{alkrassmann, andressafalcade,rafa.rjardim, roseclea.medina\}@gmail.com, \\ berchteinf.ufrgs.br
}

\begin{abstract}
This paper presents a Systematic Review of Literature (SRL) of brazilian Thesis and Dissertations that have discussed the construction or use of $3 D$ Virtual Worlds in education. The searches were conducted at the Brazilian Digital Library of Thesis and Dissertations (BDTD), where 22 researches were found. As a result, it was obtained an overview of what has already been researched in the area, indicating the main characteristics of the studies, such as origin, platforms, types of research, objectives, target audiences, methods and results, elucidating suggestions of research gaps to be de filled out.
\end{abstract}

Resumo. Este artigo apresenta uma Revisão Sistemática de Literatura (RSL) de Teses e Dissertações brasileiras que discorreram sobre a construção ou o uso de Mundos Virtuais 3D na educação. As buscas foram realizadas junto à Biblioteca Digital Brasileira de Teses e Dissertações (BDTD), onde foram encontrados 22 pesquisas. Como resultado obteve-se um panorama do que já foi pesquisado na área, com a indicação das principais características dos estudos, como origem, plataformas, tipos de pesquisa, objetivos, públicosalvo abordados, métodos e resultados, elucindando sugestões de lacunas de pesquisa a serem preenchidas.

\section{Introdução}

Uma das áreas da Informática na Educação (IE) em ascensão se refere ao desenvolvimento de ambientes capazes de proporcionar imersão aos conteúdos, com a crescente difusão do uso de tecnologias como Realidade Virtual (RV), Realidade Aumentada (RA) e dos Mundos Virtuais tridimensionais (MV3D). Os MV3D, além de oferecerem uma combinação de ferramentas de simulação, proporcionam a oportunidade de comunicação e colaboração entre os seus usuários (Herpich; Tarouco, 2016).

No entanto, a adoção efetiva de MV3D não tem ocorrido de forma mais ampla, como previsto por alguns especialistas (Yoon; George, 2013; Gregory al., 2015). Nesse sentido, para alavancar e ampliar estudos na área, emerge a necessidade de que pesquisadores mostrem as melhores práticas e resultados obtidos, destacando as contribuições desses ambientes para a educação. 
No Brasil, observa-se que grande parte dos artigos acadêmicos limita-se a mostrar uma funcionalidade ou a discutir um tópico específico, estando, geralmente, relacionados ou inseridos em pesquisas maiores, como Dissertações de Mestrado ou Teses de Doutorado que, por sua vez, aprofundam-se no assunto e apresentam as pesquisas por completo. Partindo desse pressuposto, para atingir o propósito de obtenção de um panorama do cenário acadêmico brasileiro sobre MV3D educacionais, esta RSL considera Teses e Dissertações indexadas na Biblioteca Digital Brasileira de Teses e Dissertações (BDTD).

A BDTD tem por objetivo integrar, em um único portal, os sistemas de informação de Teses e Dissertações existentes no País e disponibilizar para os usuários um catálogo nacional, possibilitando uma forma única de busca e acesso a esses documentos (BDTD, 2017). Conta atualmente com mais de 470 mil registros de 144 Instituições de Ensino Superior (IES), destacando-se, assim, como uma das maiores iniciativas para a disseminação e visibilidade de pesquisas brasileiras.

O artigo está estruturado da seguinte forma: na seção 2 são apresentados o referencial teórico e os trabalhos relacionados, de forma a contextualizar e embasar a pesquisa; a seção 3 apresentada o método de pesquisa; a seção 4 apresenta a análise e discussão dos resultados, finalizando na seção 5 com as conclusões e trabalhos futuros.

\section{Referencial teórico e trabalhos relacionados}

Os MV3D podem ser considerados representações fieis de ambientes reais ou fictícios, criados para convivência e comunicação entre pessoas, representadas por avatares, que realizam ações e interagem entre si (Backes, 2012). Holden et al. (2010) afirmam que a utilização de MV3D em ambientes educativos pode proporcionar a familiarização com o conteúdo através de práticas, demonstrações, atividades de tomada de decisão e abordagem centrada no estudante.

Nesse sentido, justifica-se a ampliação de pesquisas sobre o assunto, visando contribuir com as tendências na área de IE. Pressupõe-se que é importante contextualizar as pesquisas para a realidade em que se vive, para a partir dela verificar onde mais urge avançar. Assim, neste estudo buscou-se por lacunas e problemas relativos à adoção e implementação de MV3D no Brasil, para aprimorar o escopo de uma proposta de Tese que almeja dar continuidade à exploração desses ambientes em prol da aprendizagem.

No decorrer desta RSL verificou-se a existência de certa divergência no que se refere ao conceito de Mundos Virtuais. Na busca realizada na BDTD, muitos trabalhos retornados abordaram espaço cibernético, Internet, redes sociais ou comunidades virtuais, definindo estes ambientes como MV. A maioria desses trabalhos são anteriores a 2003, quando se iniciou uma maior utilização dos MV como ambientes 3D imersivos, com o surgimento do Second Life. Assim, torna-se importante destacar que, no âmbito deste estudo, definem-se MV como ambientes tridimensionais (MV3D) representativos do mundo real, podendo estes atingir diferentes níveis de realidade, de acordo com seus propósitos e interesses (Ávila, 2016). Vale ressaltar que ambientes totalmente imersivos como CAVEs (Cave Automatic Virtual Environment), apesar de também poderem ser considerados MV3D, fogem ao escopo da pesquisa.

Nunes et al. (2016) realizaram uma RSL acessando 13 bases de dados nacionais e internacionais, entre as quais as das revistas Journal of Virtual Worlds Research 
(JVWR) e Computers \& Education, chegando a um resultado de 58 artigos. Os autores mostraram que houve um crescimento de publicações na área entre os anos de $2010 \mathrm{e}$ 2013, apresentaram uma análise da diversidade de teorias educacionais com que se fundamentaram as abordagens e destacaram oportunidades futuras.

Na pesquisa de Oliveira et al. (2016) foram selecionados 61 documentos para análise utilizando técnicas de mineração de texto, visando disseminar e encorajar a autoria em MV3D. Os autores concluíram que existem vários desafios a serem explorados, que vão desde disciplinas e conteúdos que ainda não foram abordados e abordagens que considerem a autoria multi-usuário.

Reisoglu et al. (2017) conduziram uma pesquisa empírica a qual denominaram "meta revisão", examinando um total de 167 estudos. Entre as conclusões os autores verificaram que estudos de caso e pesquisas quasi-experimentais foram as mais comuns, e que o tamanho das amostras ficou abaixo 100 para a maioria dos estudos. Além disso, constataram que o aprendizado de línguas e ciências foram os tópicos mais abordados, e que as estratégias de aprendizagem colaborativa e baseada na exploração foram as mais utilizadas.

Este estudo diferencia-se dos demais devido ao escopo restrito a Teses e Dissertações, consideradas pesquisas mais completas e profundas, e em um cenário exclusivamente brasileiro, tendo a seguinte questão de pesquisa como norteadora:

- Qual o panorama de Teses e Dissertações brasileiras que abordam a temática de MV3D voltados à educação?

Além disso, outro diferencial deste trabalho consiste em contabilizar e discorrer sobre um maior número de aspectos dos analisados nos estudos mencionados, que configuram-se como questões secundárias de pesquisa: a) quais as origens dos trabalhos desenvolvidos; b) quais as plataformas utilizadas; c) quais os tipos de pesquisa desenvolvidas; d) quais os públicos-alvo abordados; e) quais os principais objetivos; f) quais as formas adotadas para viabilizar os métodos; g) quais as formas de avaliação; e h) quais os principais resultados encontrados.

\section{Método de Pesquisa}

No intuito de responder à questão de pesquisa, uma string de busca foi formulada e inserida no site da BDTD, utilizando-se os termos "virtual worlds" em uma primeira busca e "mundos virtuais" em uma segunda busca, definindo-se que estes poderiam ser localizados em todos (quaisquer) os campos dos registros. Optou-se por inserir os termos em inglês e português visando evitar que trabalhos fossem excluídos por não estarem indexados em uma dessas línguas. Também foi optado por não inserir o termo "educação" ou "education" na busca inicial para que todos os trabalhos envolvendo a temática de MV3D fossem considerados em um primeiro momento. A coleta de dados ocorreu no mês de abril de 2017.

Diante do corpus de pesquisas obtido, a RSL executou-se em duas etapas:

Etapa 1 - Seleção: Esta etapa consistiu na leitura dos resumos das pesquisas, fazendo a seleção daquelas que se enquadraram nos Critérios de Inclusão (CI) e passaram pelos Critério de Exclusão (CE).

Etapa 2 - Extração: Nesta etapa procedeu-se à leitura completa das pesquisas 
VI Congresso Brasileiro de Informática na Educação (CBIE 2017)

Anais do XXVIII Simpósio Brasileiro de Informática na Educação (SBIE 2017)

selecionadas, fazendo novamente um confrontamento com CI e CE. Os Critérios de Inclusão (CI) e Critérios de Exclusão (CE) utilizados nas etapas da pesquisa são elencados na Tabela 1.

Tabela 1. Critérios de inclusão (Cl) e de exclusão (CE).

\begin{tabular}{|c|c|}
\hline Critérios de Inclusão (CI) & Critérios de Exclusão (CE) \\
\hline $\begin{array}{l}\text { CI 1: A pesquisa aborda a temática de Mundos } \\
\text { Vïrtuais no âmbito educacional. }\end{array}$ & $\begin{array}{l}\text { CE 1: O texto completo da pesquisa não está } \\
\text { disponível. }\end{array}$ \\
\hline $\begin{array}{l}\text { CI 2: A pesquisa aborda o processo de } \\
\text { desenvolvimento, aplicação, utilização ou } \\
\text { análise/avaliação de Mundos Virtuais no âmbito } \\
\text { educacional. }\end{array}$ & $\begin{array}{l}\text { CE 2: A pesquisa não está situada ou não possui } \\
\text { foco no âmbito educacional, sendo direcionada } \\
\text { a outras áreas como saúde, psicologia, } \\
\text { engenharia, design, computação, etc. }\end{array}$ \\
\hline \multirow[t]{2}{*}{$\begin{array}{l}\text { CH3: A pesquisa considera Mundos Virtuais como } \\
\text { ambientes tridimensionais representativos do } \\
\text { mundo real. }\end{array}$} & $\begin{array}{l}\text { CE-3: A pesquisa traz conceitos diferentes de } \\
\text { Mundos Virtuais, como comunidades virtuais } \\
\text { ou redes sociais, ou utiliza os termos da string } \\
\text { de busca somente como referência. }\end{array}$ \\
\hline & $\begin{array}{l}\text { CE-4: A pesquisa aborda diferentes tipos de } \\
\text { Mundos Virtuais tridimensionais, como os } \\
\text { totalmente imersivos CAVEs. }\end{array}$ \\
\hline
\end{tabular}

A Figura 1 apresenta uma síntese da execução da RSL, onde é possível verificar que na busca inicial obteve-se um total de 235 trabalhos. Entretanto, constatou-se que 37 destes estavam repetidos, pois apareceram nas duas buscas realizadas (em inglês e em português). Dessa forma, 198 estudos únicos foram considerados na Etapa 1 (leitura dos resumos). Para a Etapa 2 (leitura completa) restaram apenas 22 pesquisas que corresponderam à temática buscada. Os trabalhos em sua maioria foram excluídos pelos CE 2, 3 e 4, sendo que nenhuma pesquisa foi desconsiderada pelo CE 1 .

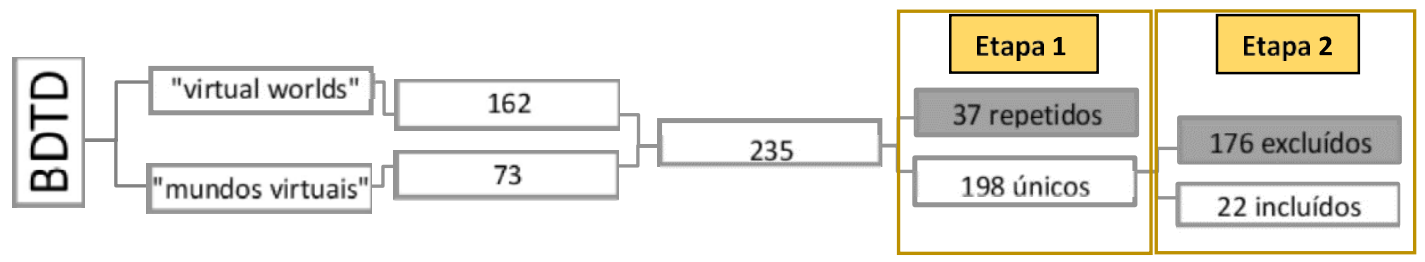

Figura 1. Síntese da RSL sobre MV3D educacionais.

Nas próximas seções são apresentados os dados resultantes da RSL, realizando a discussão do panorama de Dissertações e Teses brasileiras identificado.

\section{Análise e Discussão dos Resultados}

A análise das 22 pesquisas de Mestrado e Doutorado pretende fornecer informações úteis aos pesquisadores brasileiros da área, sobre tendências e lacunas de pesquisa. Almeja-se com isto a possibilidade de observar onde já se concentraram os estudos primários sobre os efeitos de MV3D na educação, sugerindo aspectos que ainda carecem desenvolvimento e discussão.

Dos 22 estudos que compuseram a amostra obteve-se quatro Teses de Doutorado (18\%) e 18 Dissertações de Mestrado (82\%). Entre as instituições originárias destas pesquisas destacam-se Unisinos (5), Universidade Federal do Rio Grande do Sul (UFRGS) (4), Universidade Federal de Santa Maria (UFSM) (3) e Universidade Federal de Santa Catarina (UFSC) (2). As demais instituições são a proveniência de 
apenas uma pesquisa cada. Com esta informação é possível inferir que a maioria dos estudos é originário da região Sul do Brasil (15), com destaque para estado do Rio Grande do Sul, com 13 pesquisas, sendo 3 destas Teses de Doutorado. Esta constatação corrobora a pesquisa de Oliveira et al. (2016), quanto às pesquisas nacionais estarem concentradas na regiãoSul.

Sobre o quantitativo de pesquisas realizadas por ano, na Figura 2 observa-se que entre os anos de 2012 e 2014 os estudos aumentaram, saindo de uma média de 1,5 para uma média de 4,5 pesquisas por ano, com o ápice chegando no ano de 2012, onde um total de 8 pesquisas foram produzidas.

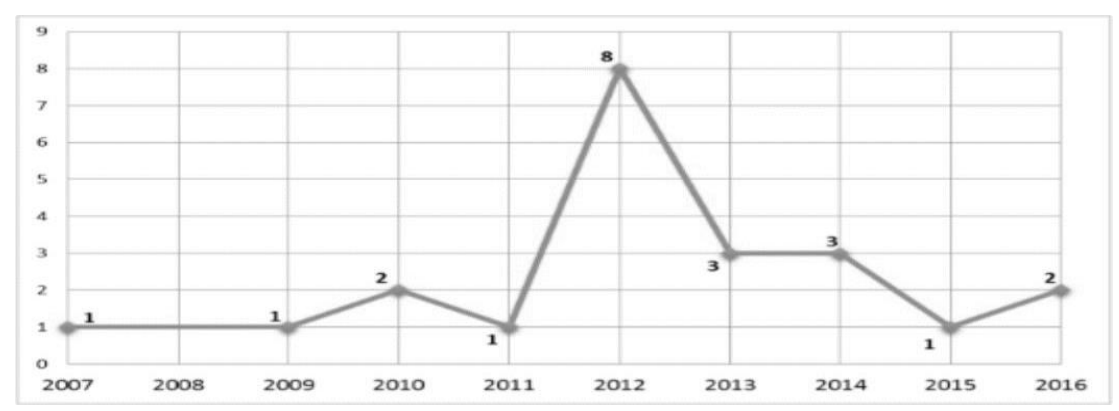

Figura 2. Quantidade de pesquisas da amostra por ano.

Esse crescimento entre os anos de 2012 a 2014 vai em consonância com o identificado no trabalho de Nunes et al. (2016), que constatou um aumento a nível mundial de artigos entre os anos de 2010 a 2013. Porém, apesar de em 2015 ter sido produzida apenas uma pesquisa no Brasil (Dissertação), a expectativa é de que este número cresça gradualmente nos próximos anos, tendo em vista a quantidade de pesquisas atualmente em desenvolvimento de que os autores têm conhecimento.

Quanto aos Programas de Pós-Graduação (PPG) de origem das pesquisas, observou-se que boa parte dos trabalhos provêm diretamente da Educação (6) e da Informática/Computação/Tecnologias da Informação e Comunicação (TIC) (6). Na sequência, Administração (4), Engenharia (4) e Interdisciplinar (2). Nesta última categoria estão os PPG em Informática na Educação (PPGIE) da UFRGS (1) e em Educação em Ciências da Universidade Federal do Rio Grande (FURG)(1).

No que se refere às plataformas de desenvolvimento de MV3D utilizadas, destaca-se com maioria a Second Life (11), em concordância com o que também foi identificado nas pesquisas de Nunes et al. (2016) e Reisoglu et al. (2017). Logo em seguida, as plataformas OpenSimulator (7) e Open Wonderland (1). Este fato pode estar ligado à facilidade do Second Life enquanto um serviço disponível na internet, não necessitando de servidores próprios. Em contrapartida, OpenSimulator e Open Wonderland são plataformas open source, livres de pagamentos e limitações de uso, mas que requerem maior esforço técnico para instalação e configuração.

Uma pesquisa fez uso de mais de uma plataforma no mesmo estudo: Second Life e OpenSimulator. Ávila (2016) em sua Tese conduziou diferentes experimentos, ofertando cursos de capacitação para professores da educação básica em cada uma das plataformas. Além disso, dois trabalhos fizeram uso de plataformas diferentes das convencionalmente utilizadas: Active Worlds, um ambiente web 3D criado em 1995 pela Activeworlds Inc., e Plataforma Toogle, proposta para implementação de sistemas físicocibernéticos. 
Quanto aos demais itens analisados (tipos de pesquisa, público-alvo, objetivos, formas de viabilização dos métodos, avaliação/validação e principais resultados encontrados) verificou-se a existência de certa similaridade entre os trabalhos. Assim, para facilitar a construção do panorama almejado, a análise foi realizada por meio de agrupamentos dos estudos em categorias exclusivas (apenas um trabalho porcategoria).

No tocante aos tipos de pesquisas realizadas, verifica-se na Tabela 2 que o Estudo de Caso ocorreu na maioria dos trabalhos (13), o que também foi predominante na revisão de literatura realizada por Reisoglu et al. (2017). Esta forma de pesquisa é típica em áreas mais novas, nas quais não se dispõe de grandes bases de dados para testar teorias, como é o caso dos MV3D, sendo também interessante para avaliar o comportamento de usuários quando utilizam ambientes propostos.

Tabela 2. Tipos de pesquisa realizadas nos estudos da amostra.

\begin{tabular}{|c|c|c|}
\hline Item & Tipo de pesquisa & Quantidade \\
\hline $\mathbf{1}$ & Estudo de Caso & 13 \\
\hline $\mathbf{2}$ & Aplicada e Descritiva & 2 \\
\hline $\mathbf{3}$ & Exploratória & 2 \\
\hline $\mathbf{4}$ & Múltiplos Métodos & 2 \\
\hline $\mathbf{5}$ & Experimental & 1 \\
\hline $\mathbf{6}$ & Explicitação do discurso Subjacente & 1 \\
\hline $\mathbf{7}$ & Método Clínico & 1 \\
\hline
\end{tabular}

Cabe ressaltar que na categoria "Múltiplos Métodos" obteve-se combinações dos métodos Estudo de Caso e pesquisa Exploratória (Voss, 2014), e desta última com pesquisa Aplicada e Descritiva (Novelli, 2013). Destaca-se que nesta análise não houve diferenciação quanto à abordagem, natureza, objetivos ou procedimentos técnicos, sendo todos estes englobados quanto ao "tipo de pesquisa".

Quanto ao público-alvo, a maioria dos trabalhos voltou-se a alunos de graduação, totalizando 9 trabalhos direcionados ou aplicados neste nível de ensino. Um dos estudos não teve aplicação com público, pois tratou da realização de testes técnicos de implementação (Santos, 2014). Esses dados são apresentados na Tabela 3 (à esquerda), que também elucida os domínios de abordagem (à direita).

Tabela 3. Público-alvo (à esquerda) e domínios de abordagem dos estudos (à direita).

\begin{tabular}{|c|c|c|c|}
\hline \multicolumn{2}{|c|}{ Público-alvo } & \multicolumn{2}{c|}{ Domínio } \\
\hline Alunos de graduação & 9 & Formação de professores & 4 \\
\hline Alunos de pós-graduação & 5 & Educação & 4 \\
\hline Alunos de educação básica & 2 & Computação & 4 \\
\hline Professores universitários & 2 & Engenharia & 3 \\
\hline Professores de educação básica & 2 & Administração & 3 \\
\hline Alunos não regulares & 1 & Ciências & 2 \\
\hline Sem aplicação com público & 1 & Física & 2 \\
\hline
\end{tabular}

No âmbito desta pesquisa considera-se domínio a área do conhecimento específica ou campo de atuação ao qual a pesquisa está direcionada. Dessa forma, identificou-se na amostra sete domínios diferentes, o que denota a diversidade de aplicações dos Mundos Virtuais com o propósito de mediação pedagógica em diferentes áreas de estudo, como, por exemplo, meio de simulação de atividades reais no caso dos trabalhos aplicados na área educacional de engenharia (Carmo, 2013; Rodrigo Silva, 2012; Schaf, 2011). 
A Tabela 4 apresenta as cinco principais categorias de objetivos de pesquisa identificados. Com esses dados nota-se que houve uma grande preocupação nos estudos da amostra em melhorar processos de ensino-aprendizagem por meio do uso de MV3D, sendo este o objetivo da maioria (10). Como exemplo é possível mencionar a Dissertação de Greis (2012), que investigou de que maneira um maior nível de interatividade e imersão, proporcionados por uma simulação construída em um MV, poderia favorecer o engajamento do aluno e ser um elemento facilitador na construção de conhecimentos sobre Física.

Tabela 4. Principais categorias de objetivos das pesquisas da amostra.

\begin{tabular}{|c|c|c|}
\hline Ordem & Objetivos de pesquisa & Quantidade \\
\hline $\mathbf{1}^{\mathbf{0}}$ & Identificar formas com que MV3D podem favorecer a aprendizagem & 10 \\
\hline $\mathbf{2}^{\mathbf{0}}$ & Entender o uso, a viabilidade ou a influência de MV3D na educação & 5 \\
\hline $\mathbf{3}^{\mathbf{0}}$ & Verificar estratégias para autoria e construção de MV3D educacionais & 4 \\
\hline $\mathbf{4}^{\mathbf{0}}$ & Possibilitar melhores formações de professores utilizando MV3D & 2 \\
\hline $\mathbf{5}^{\mathbf{0}}$ & Transpor ambiente de aprendizagem web para a tecnologia de MV3D & 1 \\
\hline
\end{tabular}

Boa parte dos estudos (5) dedicou-se a entender de que forma ocorre esta interação, averiguando a aceitação por parte de estudantes e professores. Pires (2010), por exemplo, buscou analisar de que forma a (re)criação de identidades digitais virtuais, por meio de avatares, pode superar a falta de presença do professor na educação online. Também destacaram-se estudos que objetivaram verificar meios para promover autoria e construção de MV3D na educação (4). Este é o caso das pesquisas de Ávila (2016) e Backes (2007), que apresentaram estratégias para instrumentalizar professores e ampliar o uso desses ambientes.

Sobre a forma com que os métodos de pesquisa foram viabilizados verificou-se uma dividisão em praticamente duas categorias: 12 trabalhos abordaram a "utilização de Mundos Virtuais 3D", e nove exploraram a "construção de Mundos Virtuais 3D". Ambas são abordagens práticas, a primeira requerendo um nível de conhecimento sobre programação, e a segunda requerendo um maior grau de familiariade com aspectos mais técnicos, como configurações de servidores.

Para exemplificar estas abordagens é possível mencionar as Dissertações de Mestrado de Antonio (2016), Soares (2009) e Maria (2012), que fizeram uso de ambientes já construídos na plataforma Second Life para a oferta de cursos para alunos do sexto ano do ensino fundamental, estudantes de pós-graduação, e de formação pedagógica para professores do ensino superior, respectivamente. Já Voss (2014) e Tarcila Silva (2012) optaram por construir MV3D para o ensino de Ciência da Computação, o primeiro focando na disciplina de Redes de Computadores e o segundo em Engenharia de Software. Além disso, uma Tese de Doutorado abordou a "avaliação de Mundo Virtual 3D" (Santos, 2014), focando no aspecto técnico da ferramenta em si.

Para contabilizar os tipos de avaliações que foram realizadas para validação ou testes das abordagens propostas considerou-se a principal forma de coleta de dados utilizada pelos autores das pesquisas. O meio de Entrevistas somou nove delas e Questionários foram aplicados em seis dos estudos analisados. As demais dividiram-se em Análise Empírica e de Conteúdo (3), Inspeção de Interface (3) e Desempenho do Sistema (1). 
Entrevistas e Questionários podem ser considerados boas formas de capturar a opinião dos participantes de experimentos, sendo instrumentos recorrentemente utilizados para analisar o impacto de determinada abordagem junto ao público-alvo. Observa-se que estes são métodos coerentes com a novidade inerente a MV3D na maioria das instituições brasileiras, de forma a obter um feedback direto dos usuários. Tatiana Silva (2012) em sua Tese fez uso de Entrevistas para entender como a introdução e o uso de MV afetam o ambiente de ensino que professores universitários estão acostumados. Já Rodrigo Silva (2012) em sua Dissertação utilizou Questionário para identificar as principais características do Second Life que poderiam potencializar o Design Educacional em cursos à distância.

As demais avaliações focaram em experiências subjetivas do próprio pesquisador, do público-alvo ou em aspectos técnicos dos sistemas. Como exemplo é possível mencionar a Dissertação de Nuernberg (2015), que aplicou um método de avaliação de Interação Humano Computador (IHC) (Inspeção de Interface) chamado percurso cognitivo, para coleta de opinião de especialistas sobre a construção de uma secretaria acadêmica em MV3D.

Para finalizar, buscou-se elucidar as principais contribuições e resultados alcançados ao final das pesquisas realizadas. Na Tabela 5 é possível observar que sete trabalhos apresentaram a obtenção de "resultados positivos de aprendizagem ou boa aceitação dos alunos utilizando MV3D". Sendo assim, em se tratando de Teses e Dissertações sobre Mundos Virtuais 3D no Brasil, pode-se concluir que boa parte dos estudos desenvolvidos alcançou em seus resultados a inferência ou diagnóstico de aspectos positivos junto aos estudantes.

Tabela 5. Principais categorias de resultados encontrados nas pesquisas da amostra.

\begin{tabular}{|c|c|c|}
\hline Item & Principais resultados encontrados & Quantidade \\
\hline $\mathbf{1}$ & Resultados positivos na aprendizagem / aceitação dos alunos utilizando MV3D & 7 \\
\hline $\mathbf{2}$ & Boa aceitação de MV3D pelos professores & 5 \\
\hline $\mathbf{3}$ & Viabilidade técnica de construção de MV3D na educação & 5 \\
\hline $\mathbf{4}$ & Potencial de MV3D para uso educacional & 5 \\
\hline
\end{tabular}

Para exemplificar o constatado, a pesquisa de Greis (2012) concluiu que a imersão do aluno em um MV3D relacionado à disciplina de Física favorece o engajamento dos alunos pois o ambiente oferece um nível de realismo, com a possibilidade de vivenciar uma situação verossímil em uma simulação educacional. A Dissertação de Pessoa (2013), por sua vez, mostrou indícios de que um laboratório imersivo contribuiu para o aumento da motivação dos alunos, dadas as características de interação, múltiplas perspectivas, fidelidade e relevância da lição no ambiente real.

Também foi identificado como resultado das pesquisas: "boa aceitação de MV3D pelos professores" (5); a afirmação de "viabilidade técnica para a construção de MV3D na educação" (5); e que MV3D apresentam "potencial para uso educacional" (5). O estudo de Ávila (2016), enquadrado nesta última categoria, concluiu que é viável a exploração desses ambientes para atender estudantes da educação básica e revelou que professores deste nível têm condições de tornarem autores em MV.

\section{Conclusão}

A utilização de Mundos Virtuais 3D (MV3D) para a geração de conhecimentos em diferentes áreas vem sendo ampliada, principalmente devido às possibilidades trazidas 
por esses ambientes, como imersão aos conteúdos, interação síncrona de usuários e simulação de situações reais, facilitando abordagens ativas e atividades de experimentação. Nesse sentido, faz-se pertinente verificar o que está sendo desenvolvido na área a fim de identificar até onde já foi possível avançar e as limitações da aplicabilidade educacional.

Este estudo buscou contribuir com a temática e complementar as pesquisas de Nunes et al. (2016), Oliveira et al. (2016) e Reisoglu et al. (2017), que também analisaram o estado da arte sobre MV3D voltados à educação. Porém, nesta pesquisa focou-se na análise das produções desenvolvidas em Programas de Pós-Graduação Strictu Sensu no Brasil. Para tanto foi realizada uma Revisão Sistemática da Literatura (RSL) junto à Biblioteca Digital Brasileira de Teses e Dissertações (BDTD), que resultou em uma amostra de 22 pesquisas que corresponderam ao escopo buscado, sendo 18 Dissertações de Mestrado e quatro Teses de Doutorado.

Como resultado observou-se que os estudos concentraram-se principalmente no Rio Grande do Sul, entre os anos de 2012 e 2014, advindas de PPG's como Educação e Informática, e com predominância de uso da plataforma Second Life. A pesquisa do tipo Estudo de Caso foi realizada pela maioria dos trabalhos, tendo como público-alvo em maior parte alunos de graduação. $\mathrm{O}$ domínio de aplicação variou principalmente entre as áreas de Computação, Educação e Formação de Professores e o principal objetivo almejado foi identificar as formas com que MV3D pode favorecer a aprendizagem.

A maior parte dos estudos analisados fez uso de MV3D pré-construídos, evitando a necessidade de lidar com configurações de servidores. A coleta de dados ocorreu primordialmente por meio de Entrevistas e entre os principais resultados encontrados está a boa aceitabilidade desses ambientes por parte de professores e alunos, a indicação de que o desenvolvimento e a implementação dos mesmos é considerada viável, e a obtenção de indícios de que a utilização de MV3D de fato enseja a aprendizagem dos estudantes.

Os resultados elucidados nesta pesquisa podem guiar a definição de temas para novas Dissertação de Mestrado e Teses de Doutorado que circundem a temática. Conclui-se que há uma lacuna de pesquisas de abordagem quantitativa e realizadas fora dos domínios de Educação e Informática, que são geralmente as áreas de origem dos estudos. Observa-se também a necessidade de trabalhos que abordem outros níveis de ensino além da graduação. Não foi identificada nenhuma pesquisa com enfoque na análise de aspectos afetivos com o apoio da Computação Afetiva, como, por exemplo, a influência de agentes pedagógicos afetivos nesses ambientes. Em concordância com Oliveira et al. (2016), também observa-se a carência de estudos que abordem a autoria em MV3D, o que foi constatado somente na pesquisa de Ávila (2016), mas que colocou somente o professor como autor.

Como trabalhos futuros pretende-se ampliar esta pesquisa para incluir e gerar um comparativo dos estudos brasileiros com outros países, no intuito de identificar o patamar dessa temática em nível mundial e a posição do Brasil neste cenário, indicando possibilidades para alvancar as pesquisas na área.

\section{Referências}

Antonio, C. P. (2016). Mundos virtuais 3D integrados à experimentação remota. 
VI Congresso Brasileiro de Informática na Educação (CBIE 2017)

Anais do XXVIII Simpósio Brasileiro de Informática na Educação (SBIE 2017)

Dissertação de Mestrado. Programa de Pós-Graduação em Tecnologias da Informação e Comunicação. Universidade Federal de Santa Catarina(UFSC).

Ávila, B. G. (2016). Formação docente para a autoria nos mundos virtuais: uma aproximação do professor às novas demandas tecnológicas. Tese de Doutorado. Programa de Pós-Graduação em Informática na Educação (PPGIE), Universidade Federal do Rio Grande do Sul (UFRGS).

Backes, L. (2007). Mundos virtuais na formação do educador: uma investigação sobre os processos de autonomia e de autoria. Dissertação de Mestrado. Programa de Pósgraduação em Educação. Universidade do Vale do Rio dos Sinos(UNISINOS).

Backes, L. (2012). As manifestações da autoria na formação do educador em espaços digitais virtuais. Revista de Educação, Ciência e Cultura. vol. 17, nº. 2, jul./dez.

BDTD. (2017). Biblioteca Digital Brasileira de Teses e Dissertações. Instituto Brasileiro de Informação em Ciência e Tecnologia (IBICT). Disponível em: http://bdtd.ibict.br/vufind/. Acesso em: 11 de abril de 2017.

Carmo, F. M. D. (2013). Mundo virtual 3D em plataforma aberta como interface para ambientes de aprendizagem. Dissertação de Mestrado. Programa de Pós-graduação em Engenharia, Universidade de São Paulo (USP).

Herpich, F.; Tarouco, L. M. R. (2016). Engajamento de usuários em mundos virtuais: uma análise teórica-prática. Revista Novas Tecnologias na Educação (RENOTE), v. 14, n. 1 .

Holden, J.T; Westfall, P. J. L.; Emeriti, C. (2010) An Instructional Media Selection guide for distance learning implications for bended learning featuring an introduction to virtual worlds. In: USDLA United States Distance Learning Association. Second Edition.

Greis, L. K. (2012). Mundos virtuais na educação: a interatividade em simulações de fenômenos físicos. Dissertação de Mestrado. Programa de Pós-Graduação em Educação. Universidade Federal do Rio Grande do Sul (UFRGS).

Gregory, S. S, Scutter, S., Jacka, L., Mcdonald, M., Farley H., Newman, C. (2015). "Barriers and Enablers to the Use of Virtual Worlds in Higher Education: An Exploration of Educator Perceptions, Attitudes and Experiences", Educational Technology \& Society, 18 (1), 312.

Maria, S. A. A. (2012). Proposta de formação continuada para docentes da educação superior no metaverso second life. Dissertação de Mestrado. Programa de PósGraduação em Educação. Universidade Federal do Rio Grande do Sul (UFRGS).

Noveli, M. (2013). A virtualização de processo com o uso de mundos virtuais: um estudo do processo ensino-aprendizagem a partir da perspectiva dos participantes do processo. Tese de Doutorado. Escola de Administração de Empresas, Fundação Getulio Vargas (FGV).

Nuernberg, J. (2015). 3DVSAS: 3D VIRTUAL SPACE OF THE ACADEMIC SECRETARIAT. Dissertação de Mestrado. Programa de Pós-Graduação em Informática. Universidade Federal de Santa Maria (UFSM).

Nunes, F., B.; Herpich, F.; Paschoal, L. N.; Tarouco, L. M. R.; Lima, J. V. (2016). Systematic Review of Virtual Worlds applied in Education. V Congresso Brasileiro 
de Informática na Educação (CBIE).

Oliveira, L. C., Espíndola, D. B., Amaral, M. A., Barwaldt, R., \& da Costa Botelho, S. S. (2016). Authorship Methodological Artifact Applied to Virtual Worlds for Education. Brazilian Journal of Computers in Education, 24(03), 97.

Pessoa, F. M. M. (2013). Aprendizagem imersiva em mundos virtuais. Dissertação de Mestrado. Programa de Pós-graduação em Ciência da Computação. Universidade Federal de Pernambuco (UFPE).

Pires, D. T. (2010). Educação Online em Metaverso: a mediação pedagógica por meio da telepresença e da presença digital virtual via avatar em Mundos Digitais Virtuais em 3 Dimensões. Dissertação de Mestrado. Programa de Pós-graduação em Educação. Universidade do Vale do Rio dos Sinos (UNISINOS).

Reisoglu, I., Topu, B., Yilmaz, R., Yilmaz T. K., Goktas, Y. (2017). 3D virtual learning environments in education: a meta-review. Asia Pacific Education Review, 18(1), 81-100.

Santos, R. A. P. dos. (2014). Ambientes físico-virtuais de aprendizagem. Tese de Doutorado. Programa de Pós-graduação em Educação em Ciências. Universidade Federal do Rio Grande (FURG).

Schaf, F. M. (2011). Arquitetura modular para ambientes virtuais de ensino de automação com suporte à realidade mista e colaboração. . Tese de Doutorado. Programa de Pós-Graduação em Engenharia Elétrica. Universidade Federal do Rio Grande do Sul (UFRGS).

Silva, Rodrigo. G. da. (2012). O potencial educacional dos mundos virtuais tridimensionais. Dissertação de Mestrado. Programa de Pós-Graduação em Engenharia e Gestão do Conhecimento. Universidade Federal de Santa Catarina (UFSC).

Silva, Tarcila. G. (2012). Jogos Sérios em Mundos Virtuais: uma abordagem para o ensino- aprendizagem de Teste de Software. Dissertação de Mestrado. Programa de Pós- Graduação em Informática. Universidade Federal de Santa Maria (UFSM).

Silva, Tatiana. M. B. da (2012). A introdução de metaversos no processo de ensino: Fazendo sentido de mundos virtuais no contexto organizacional do ensino superior. Tese de Doutorado. Pontifícia Universidade Católica do Rio de Janeiro (PUC-RJ).

Soares, A. C. (2009). O Second Life na educação. Dissertação de Mestrado. Programa de Pós-graduação em Educação. Universidade de Brasília (UnB).

Yoon, T. E., George J. F. (2013) "Why aren’t organizations adopting virtual worlds?" Computers in Human Behavior, Vol. 29, Issue 3.

Voss, G. (2014) TCN5-Teaching Computer Networks in a Free Immersive Virtual Environment. Dissertação de Mestrado. Programa de Pós-Graduação em Informática. Universidade Federal de Santa Maria (UFSM). 